\title{
Kräuterbücher (1)
}

Sammelleidenschaft und Forschungseifer: Zwei Gelehrte aus verschiedenen Jahrhunderten auf den Spuren von Opium und Kaffee

\section{Barbara Brauckmann}

Im Nachlass des am 25. Februar 1917 in Zürich verstorbenen Pharmazeuten Carl G. Hartwich befinden sich neben Dissertationen seiner Studenten, eigenen Veröffentlichungen und Drucken anderer Forscher auch Dispensatorien, Apotheker-Taxen, Rezept-, Jahr- und Kräuterbücher sowie spagyrische oder «chymische Schriften» aus der Zeitspanne von 1520 bis 1915.

\section{Menschliche Genussmittel von Alkohol bis Tee}

Neben Pharmazeutischer Chemie, Toxikologie, Mikroskopie, ätherischen Ölen, Lebensmittelanalyse und Pharmakopöewesen standen Gewürze und Genussmittel im Fokus seines Interesses. Entsprechend handeln seine Publikationen und Vorträge nicht nur von chinesischen Birngallen, Coloquintensamenschalen, Safranverfälschung, giftigem Sternanis, Kakaobohnen, Pfeffer, Zimt oder Vanille, sondern auch von ostindischen, bolivianischen oder chilenischen Drogen, Schlafmohn (Papaver), Strychnos und Pfeilgiften [1].

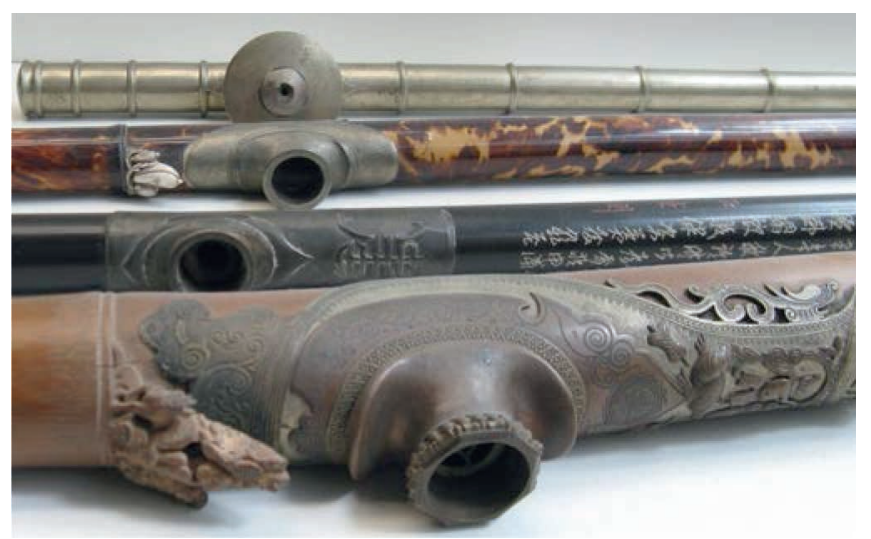

Abb. 1. Opiumpfeifen aus China aus verschiedenen Materialien gefertigt (Metall, mit Schildplattüberzug, Teebaumholz mit chinesischen Schriftzeichen, aus Bambus mit Metallverzierung). Pharmakognostische Sammlung des Instituts für Pharmazeutische Wissenschaften (IPW) der ETH Zürich; Nachlass von Prof. Carl Hartwich (Foto: Öffentlichkeitsarbeit des Departementes Chemie und Angewandte Biowissenschaften, ETH Zürich).
1892 habilitierte er sich über «Die Bedeutung der Entdeckung von Amerika für die Drogenkunde» an der Technischen Hochschule zu Braunschweig und wurde nur wenige Monate danach als Professor der Pharmakognosie, Pharmazeutischen Chemie und Toxikologie an das Eidgenössische Polytechnikum nach Zürich berufen. Die Zürcher Universität ernannte ihn später zum Doktor medicinae honoris causa [1].

Hartwich wurde am 26. März 1851 in Tangermünde in der preussischen Provinz Sachsen geboren. Er absolvierte das Gymnasium in Stendal sowie eine dreijährige Lehrzeit in der väterlichen Apotheke und hörte ab 1876 drei Semester lang an der Universität Berlin Vorlesungen zur Botanik und Chemie. Nach dem pharmazeutischen Staatsexamen, einer Dienstzeit als Militärapotheker und einer Tätigkeit als Fabrik-Chemiker verkaufte er 1891 die vom Vater vererbte Apotheke und promovierte in Bern mit dem «Beitrag zur Kenntnis der Strophanthus- und einiger mit denselben verwandter Samen».

Als Hauptwerk des Wissenschaftlers gilt das 1911 erschienene Buch «Die Menschlichen Genussmittel - Ihre Herkunft, Verbreitung, Geschichte, Anwendung, Bestandteile und Wirkung». Hier beschreibt er die nach jahrzehntelangem Quellenstudium und eigenen Analysen erzielten Erkenntnisse zu Opium, Hanf, Koka, Alkohol, Kaffee, Tee, Schokolade, Tabak, Kat und Betel. Dabei bezieht er die geschichtlichen, handelspolitischen und ethnographischen Aspekte ein.

\section{Hartwich 1898: «Opium, Genussmittel im niedrigsten Sinn»"}

Offensichtlich faszinierte ihn das Opium, denn einige seiner Publikationen sind dem Rauchopium oder den beim Opiumrauchen wirksamen Stoffen gewidmet (Abb. 1). Der Bericht «Das Opium als Genussmittel» im Neujahrsblatt 1898 [2] der Naturforschenden Gesellschaft in Zürich umfasst mehr als 40 Druckseiten. Hartwich,

\section{KARGER}

Fax +497614520714 Information@Karger.con www.karger.com
Dr. Barbara Brauckmann

Öffentlichkeitsarbeit, Departement Chemie und Angewandte Biowissenschaften ETH Zürich

HCI J 57.2, Vladimir-Prelog-Weg 10, 8093 Zürich, Schweiz

brauckmann@chem.ethz.ch 
inzwischen Vorstand der Pharmazeutischen Abteilung der Eidgenössischen Technischen Hochschule, betont darin:

\begin{abstract}
«Das Opium nimmt unter den Genussmitteln eine etwas eigentümliche und abgesonderte Stellung ein. Es ist nur Genussmittel im engsten oder, sagen wir, niedrigsten Sinne. Während bei den andern aber wieder die Verwendung als Genussmittel an der Spitze steht und die als Heilmittel eine mehr nebensächliche oder doch vielleicht nicht ursprüngliche ist, hat man, soviel wir wissen, das Opium lange Zeit nur als Heilmittel verwendet und ist vielleicht erst später dazu gelangt, es auch für Genusszwecke in Gebrauch zu nehmen» [2].
\end{abstract}

In der Mitte seiner Ausführungen zitiert er eine Passage aus den Reisebeschreibungen des 1535 als Sohn eines Kaufmanns zu Augsburg geborenen Arztes Leonhart Rauwolf, der ihm im Rahmen seiner Forschung zu diesem Thema offensichtlich nahestand:

\begin{abstract}
«Nit minder findet man allda (nämlich im Bazar von Aleppo) auch des gesaffts von Apoteckern Opium, den jnnwonern aber Ofiun genennet, welchen die Türcken, Moren, Persae, und andere mehr völcker einzunemmen pflegen, nit allein in kriegen, umb die zeit, wenn sie wider jre feind sollen kempfen und streiten, jnen ein gut hertz un starcken muth zumachen: sondern auch zu zeiten des fridens, die sorgen und phantaseyen zu benemmen, oder auffs wenigst zu miltern. .... Wann nun einer oder mehr darmit also angefangen (dessen sie ohngefahrlich einer Erbis gross zunemmen pflegen), so könden sie nit wol mehr darvon lassen, es seye denn, das sie sich in ein kranckheit stürtzen, oder auffs wenigest jnen andere newe zufäll erregen wöllen, wie sie solches selb bekennen, wenn sie den einzunemmen etwan underlassen, dass sie sich alssdann sehr übel im leib befinden. Das Opium wirt maistthails genommen von köpflein, des weissen ölmagens, auff jr sprach Cascasch genennet ...»
\end{abstract}

\section{Rauwolf 1576: «vil schoene und frembde Kreutter»}

Jener Rauwolf soll gemäss seiner Biographie 1573 «von der Liebe zur Wissenschaft der Pflanzen und von dem Wunsche getrieben, die Heimathsorte der wichtigsten officinellen Pflanzen des Orients zu erkunden, mit Zustimmung und Erlaubniss der Seinigen seine grosse Reise angetreten zu haben» [3]. An verschiedenen Universitäten auf das Studium vorbereitet, erwarb er 1562 den Doktorgrad in Valence und studierte dann in Montpellier Botanik. In der Gegend seiner Aufenthaltsorte in Frankreich und Italien sammelte er über 600 Pflanzenarten und stellte daraus ein Herbarium her. Seine Rückkehr führte ihn über den Gotthard in die Schweiz, wo er Bekanntschaft mit Conrad Gesner machte. Im Jahr darauf, d.h. 1564, wurde Rauwolf seinerseits von dem niederländischen Gelehrten, Arzt und Botaniker Carolus Clusius besucht. Nach seiner Vermählung mit Regina Jung im Jahr 1565 liess Rauwolf sich als Arzt zuerst in Augsburg nieder, wo er auch einen Pflanzengarten begründete. Danach zog er nach Aichach und daraufhin nach Kempten.

Seine abenteuerliche, 3 Jahre dauernde Reise führte ihn 1573 über Lindau, Chur und den Splügen nach Mailand und über Nizza nach Marseille - und von dort nach Tripolis in Syrien. Nach einem längeren Aufenthalt in Aleppo reiste Rauwolf über Mossul und Urfa nach Bagdad und wieder zum Ausgangspunkt zurück.

Die durchmachten Gefahrensituationen überlebte er manchmal nur mit etwas Glück:

«Einigen Anfechtungen durch räuberische Kurden und seine eigenen Reisegefährten, war der gelehrte Mann, der seine Brust mit Päcken Pflanzenpapier, das er für seine Sammlungen mit sich führte, gepanzert hatte, herzhaft entgegengetreten und hatte sie nahezu ohne Schaden überstanden» $[3]$.

Am 7. September 1575 verliess er den Hafen von Tripolis, landete nach stürmischer Seefahrt in Venedig und kam am 12. Februar 1576 in Augsburg an. Hier erhielt er die Aufsicht über das Pestspital, bis er sich 1588 in den Streit über den Gregorianischen Kalender und die Berufung der Geistlichen verwickeln liess und entlassen wurde. In Linz fand er als Poliater et Ordinum Archiducatus Austriae Medicus Anstellung und begleitete später die oberösterreichischen Streitkräfte in den Türkenkrieg. Er starb 1596 (nach Coberus) bei der Belagerung von Hatvan an Dysenterie.

Der 1583 gedruckte Reisebericht «Aigentliche beschreibung der Raiss» in die Morgenländer (insbesondere Syrien, Judäa, Arabien, Mesopotamien, Babylonien, Assyrien, Armenien) von «Leonharti Rauwolfen, der Artzney Doctorn vnd bestelten Medici zuo Augspurg» (Abb. 2, 3) wurde gemäss Titel und Darlegung «nicht ohne geringe mühe vnnd grosse gefahr selbs volbracht» und beschreibt neben etlichen «mehr gar schön frembden vnd aussländischen Gewächsen ... auch andere denckwürdige sachen / die alle er auff solcher erkundiget / gesehen vnd observiert hat ...» [4].

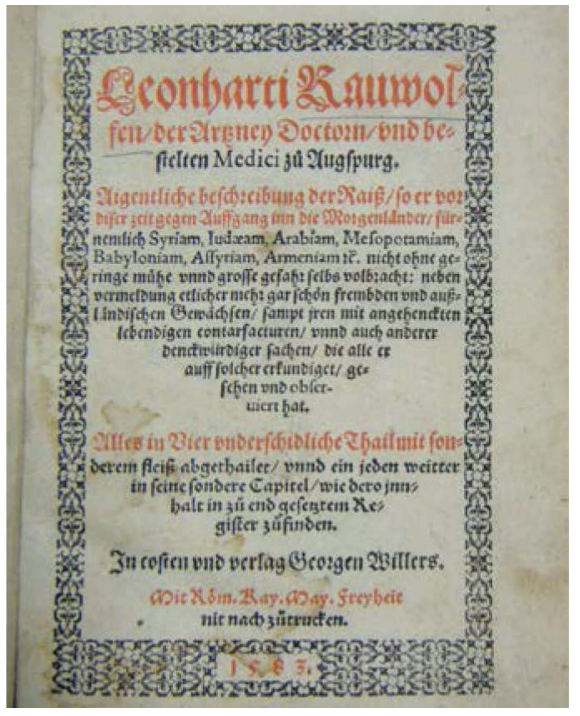

Abb. 2. Deckblatt/ Titel des Reiseberichts des Leonhart Rauwolfen: «Aigentliche Beschreibung der Raiss», 1583. Kräuterbuch der Büchersammlung aus dem Nachlass von Prof. Carl Hartwich, IPW ETH Zürich (Foto: Öffentlichkeitsarbeit des Departementes Chemie und Angewandte Biowissenschaften, ETH Zürich). 


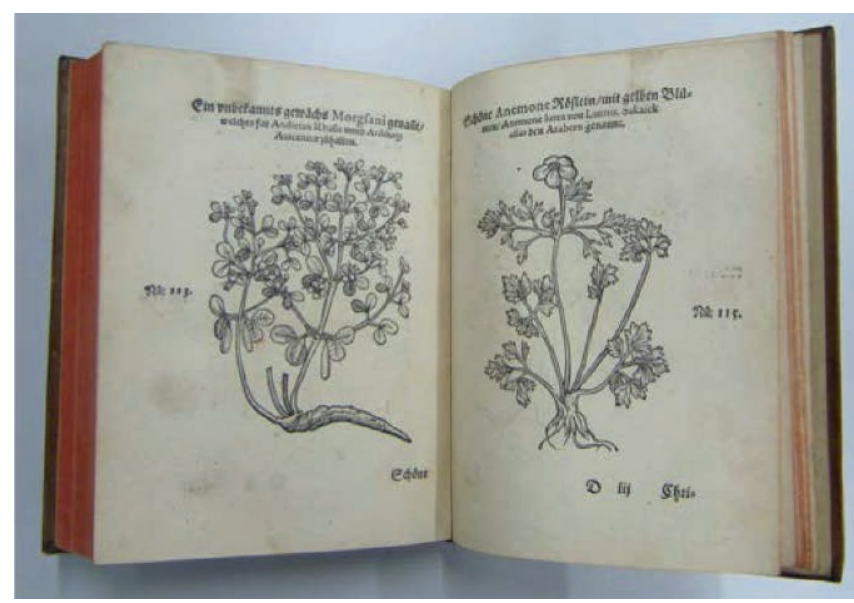

Abb. 3. Zwei Innenseiten aus dem Reisebericht des Leonhart Rauwolfen: «Aigentliche Beschreibung der Raiss», 1583. Kräuterbuch der Büchersammlung aus dem Nachlass von Prof. Carl Hartwich, IPW ETH Zürich (Foto: Öffentlichkeitsarbeit des Departementes Chemie und Angewandte Biowissenschaften, ETH Zürich).

Gemäss der Zusammenfassung von Friedrich Ratzel [3] stellt der Orientreisende medizinische Dinge, Krankheiten, Heilmittel, Bäder, Speisen und Getränke sowie die Völker, ihre Tracht und ihre Sitten in grosser Ausführlichkeit dar. Zur Natur der Gebirge und Flüsse oder zum Landschaftscharakter der durchreisten Gebiete äusserte er sich jedoch kaum. Und doch bleibt «... von den Ranunkeln und Saxifragen, die er auf dem Wege von Bregenz nach Feldkirch findet, bis zu den um Tripolis und Aleppo wachsenden Pflanzen, deren Aufzählung das ganze 4. und 9. Capitel des ersten Theiles füllt, bis zu den Bananen, dem Zuckerrohr, dem Kaffeebaum, der Dattelpalme nichts unerwähnt».

\section{Kaffee, «wie Dinten so schwarz»}

Hartwich unterhielt wie andere Forscher vor und nach seiner Zeit nicht nur eine rege Korrespondenz mit Gelehrten aus aller Welt, Freunden, Kollegen und ehemaligen Schülern sowie mit Drogenhäusern, Museen, Bibliotheken und wissenschaftlichen Instituten, sondern sammelte auch zu seinen Forschungsthemen passende Gegenstände. Über die Jahre von etwa 1890 bis $1916 \mathrm{kam}$ so für das Pharmazeutische Institut der Technischen Hochschule eine pharmakognostische Sammlung aus Gegenständen zusammen, die in ihren Ursprungsorten zum Rauchen von Opium, Tabak oder Haschisch, Kauen von Koka und Betel, zum Genuss von Tee, Maté, Kaffee, Kakao, Kola, Guarana, Sake und Kawa oder zum Gebrauch von Pfeilgiften benutzt wurden.
In einem seiner 150 verfassten Artikel berichtete der Forscher in der Zeitschrift für Untersuchung der Nahrungs- und Genussmittel sowie der Gebrauchsgegenstände (Heft 12, 18. Band, 15. Dezember 1909) über «Beiträge zur Kenntnis des Kaffees» mit Untersuchungen zu einem bolivianischen Kaffee von San Jago de Chiquitos.

2 Jahre später kommt er in seinem Buch «Menschliche Genussmittel» im Kapitel über die Geschichte des Kaffees im Orient auf Seite 309 wiederum auf den Orientreisenden aus dem 16. Jahrhundert zurück und bezeichnet diesen als ersten Europäer, der aus eigener Anschauung über den Kaffee berichtet (Abb. 4). Rauwolf schilderte in seinem Bericht, dass die Türken von Aleppo ein gutes Getränk hätten, das sie hoch halten und «Chaube» nennen:

«... das ist gar nahe wie Dinten so schwarz und in gebresten, sonderlich des Magens, gar dienlich. Dieses pflegens am Morgen fru, auch an offenen Orten, vor jedermanniglich one alles abscheuchen zu trinken aus jrdinen und pozellanischen tiefen Schälein, ...» [4].

Hartwich erwähnt in seinem Kapitel über den Kaffee aber auch Carolus Clusius, dessen «Rariorum Plantarum Historia» von 1601 sich in seinem Nachlass befindet. Andere alte, meist bebilderte Kräuter- und Botanikbücher stammen unter anderem von Petro Matthiolo, Basilius Besler, Elisabeth Blackwell, Leonharto Fuchsio, Conradi Gesneri sowie Rauwolfen Leonharti, aber auch von Albrecht von Haller, Tabernaemontanus, Christoph Jacob Trew, Otto Brunfels, Caroli Clusii, Fabri Petri Johannis, Johann Glauber, Joanne Schröders oder Friedrich Heinrich Gottlob Hayne. Auf einige der Werke wird in den folgenden Artikeln dieser Rubrik eingegangen.

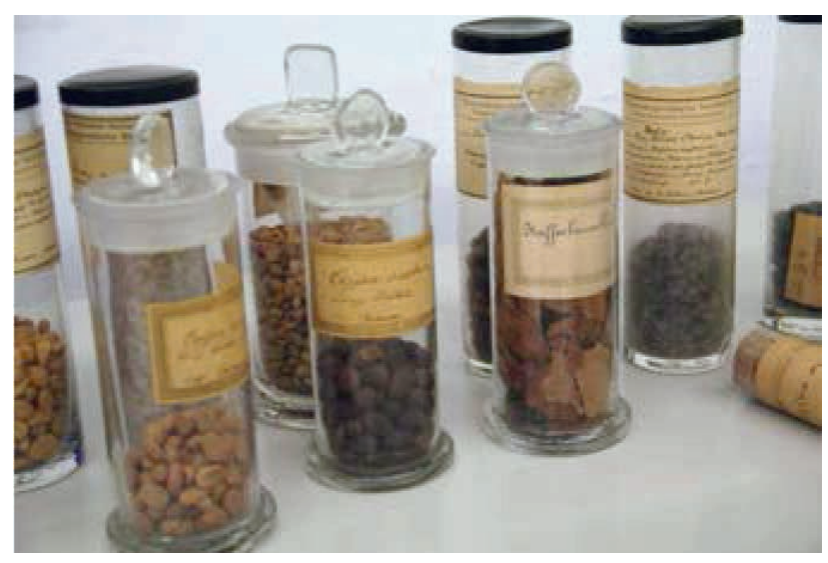

Abb. 4. Früchte und Blätter des Kaffeebaums. Pharmakognostische Sammlung des IPW der ETH Zürich; Nachlass von Prof. Carl Hartwich (Foto: Öffentlichkeitsarbeit des Departementes Chemie und Angewandte Biowissenschaften, ETH Zürich).

\section{Literatur}

1 Nachruf von Robert Eder zu Prof. C. Hartwich. Schweiz Apotheker-Zeitung 1917, Nr. 11.

2 Naturforschende Gesellschaft Zürich: Neujahrsblatt 1898. www.ngzh.ch/pdf/Neuj1898.pdf.
3 Ratzel F: «Rauwolf, Leonhard»; in: Historische Kommission bei der Bayerischen Akademie der Wissenschaften (Hrsg): Allgemeine Deutsche Biographie. Band 27. 1888, pp 462-465. Digitale Volltext-Ausgabe: http://de.wiki-
source.org/w/index.php?title=ADB:Rauwolff,_ Leonhardeoldid=1710445.

4 Rauwolf L, Salmuth H: Laugingen, Willers, 1583. http://reader.digitale-sammlungen.de/de/ fs1/object/display/bsb10180665_00005.html. 